\title{
Contracts for First-Class Classes
}

\author{
Technical Report NU-CCIS-10-04
}

\author{
T. Stephen Strickland Matthias Felleisen \\ PLT@ Northeastern University \\ \{sstrickl, matthias\}@ccs.neu.edu
}

\begin{abstract}
First-class classes add expressive power to class-based objectoriented languages. Most importantly, programmers can abstract over common scenarios with first-class classes. The addition of behavioral software contracts to a language with first-class classes poses significant challenges, however. In this paper, we present the first contract system for a programming language with first-class classes. The design has been implemented for PLT Scheme, which supports first-class classes and which implements mixins and traits as syntactic sugar. We therefore expect that our experience is also useful for languages with native mixins and/or traits.
\end{abstract}

\section{First-class Classes and Contracts}

Over the past couple of decades, programming language designers have repeatedly demonstrated the usefulness of operations on classes. For example, Bracha and Cook [7] distilled and formalized the Flavors notion of mixins ${ }^{1}$ [37]. Roughly speaking, mixins in a single-inheritance language are functions from classes to classes, adding fields and methods to the given class. Since then mixins have been the subject of numerous studies $[1,3,9,17,33]$. After noticing software engineering problems with mixins, Schärli et al. [42] added traits to Smalltalk. A trait encapsulates only behavior, that is, methods. To create a class, a programmer simultaneously derives a class from a superclass and combines it with traits via glue code that manages name clashes or other small mismatches. Both Scala [38] and Fortress [2] support traits.

Smalltalk [19], OCaml [29, 41], and PLT Scheme [15] take the final step and turn classes into first-class values. In such a world, the evaluation of an expression may produce a class. Methods or functions may consume and produce classes. Classes may store classes in fields. And most importantly, the super-class designator in a class is just an expression, meaning the super-class is computed at runtime. Equipped with this power, a programmer can add mixins and traits as "syntactic sugar" [16] but even better, a programmer can explore entirely new ways of organizing class-based programs.

PLT Scheme, like Eiffel [36] and many other object-oriented languages $[5,8,21-24,26,31,32,36,40]$, also comes with a contract system. A contract [6] is a logical assertion that governs the flow of values between distinct components. The compiler and the run-time system cooperate to monitor contracts so that they can issue an informative report when values fail to respect a contract. In PLT Scheme, a report on a contract violator both explains the nature of the violation and pinpoints the violator.

Extending contracts to first-class classes poses significant challenges. First, it demands a language for specifying contracts for

\footnotetext{
${ }^{1}$ The concept of mixins first appeared in H. I. Cannon's 1979 unpublished paper "Flavors: A non-hierarchical approach to object-oriented programming," an MIT technical report in draft.
}

classes as values. Second, when classes are first-class values, their separate roles as sources of new objects and as containers for reusable code requires rather different forms of specification than those of Eiffel [36] or even something as general as JML [27]. Third, adding contracts to first-class classes demands an implementation strategy that affects the performance of classes without contracts. Finally, PLT Scheme in particular also mixes conventional inheritance and Beta-style inheritance, complicating the addition of contracts even more.

Due to these challenges, PLT Scheme has not supported contracts for its class system, only for individual objects. Over the past year, we have designed, implemented, and experimented with a contract system that overcomes all these obstacles. Furthermore, we have extended the contract system to cope with Beta-style inheritance [28], as PLT Scheme is the first language to combine conventional inheritance with Beta-style inheritance [20]. While our practical work exploits PLT Scheme, we conjecture that the ideas apply to languages with mixins, traits, and similar features.

\section{First-class Classes in PLT Scheme}

PLT Scheme extends the Scheme programming language [43] with a class system. In this section, we briefly describe this class system; for details, we refer the interested reader to a tutorial paper [16]. We start with an example program that illustrates the Java-like features of the PLT Scheme class system. We then describe the use of classes as first-class values and show how to define mixins, one popular usage pattern. While we do not discuss traits, we encourage the reader to inspect Flatt et al.'s report to see traits as syntactic sugar for themselves.

\subsection{Simple PLT Scheme Classes}

The core of the PLT Scheme class system is similar to Java's. Figure 1 displays the skeleton of a small module that utilizes some of the basic features. The first line specifies the language of the module, here "scheme" for "PLT Scheme." The first two definitions name two new classes: web\%, ${ }^{2}$ a subclass of the root class object $\%$, and logged-web\%. The body of each class is a mixture of definitions and expressions. The expressions are collected into an initializer, which is evaluated at the end of object construction. During initialization, each class must explicitly invoke the superclass's initializer via the super-new expression.

The class web\% defines an initialization argument runs-on, which is used during object creation and defaults to a value of 8080; a public field port, which defaults to the value of runs-on; and a private field listener, which creates a TCP listener on the given port. Initialization arguments cannot be used in the bodies

\footnotetext{
${ }^{2}$ The use of $\%$ at the end of an identifier is a convention used by PLT Scheme programmers to signal that a variable stands for a class.
} 
of methods - they are only valid during the evaluation of expressions that run for object initialization, such as the default values of fields and top-level expressions. In addition, web\% contains three public methods: read-request, which parses an HTTP request from a given input port; handle-request, which performs the requested action; and serve, which accepts connections on the given port and uses the request parser and handler.

The class logged-web\% defines a public field log-file via the field form and a private method $\log$. It also uses the inherit-field form to gain direct access to the superclass's field port. The private method $\log$ is then used to implement the logging functionality in an overriding declaration of the handle-request method. The overridden definition in the superclass is available to the subclass via the super form. The third definition instantiates and then names an object of the logged-web\% class using the new form. This definition provides a different value from the default for the initialization argument runs-on. The fourth and last part is a plain expression; it invokes the serve method on server via send.

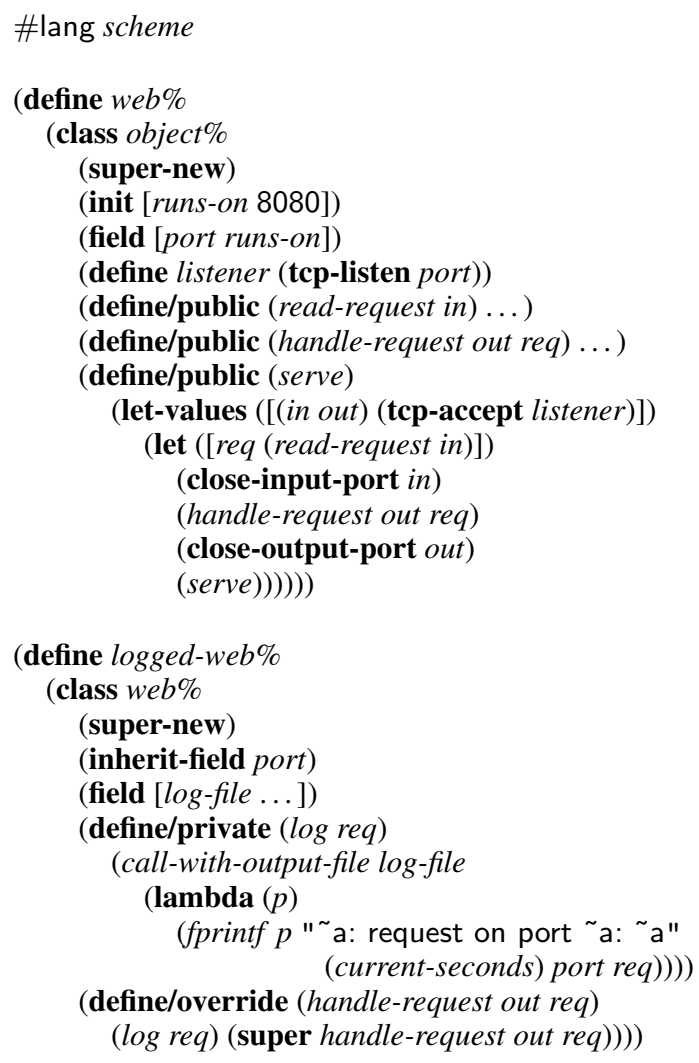

(define server (new logged-web\% [runs-on 5678]))

(send server serve)

Figure 1. A simple object-oriented program in PLT Scheme

Naturally, method definitions may refer to this, which denotes the object on which the method was invoked. Fields and methods internal to the class are directly accessible via their names, instead of accessing them via this. Examples of this convention are the uses of listener, read-request, and handle-request within the method serve in web\%. If the field or method is not defined in the same class, the programmer must declare it as an expected member of the superclass by using the inherit or inherit-field forms.

\subsection{First-class Classes and Mixins}

Classes in PLT Scheme are first-class values. Most importantly, functions may consume and produce classes. The form used to create classes is also an expression like any other in the language. Hence, classes can be created conditionally, or a single class form can be evaluated multiple times, each time resulting in a new class. Thus, the class hierarchy can be arbitrarily extended at run-time.

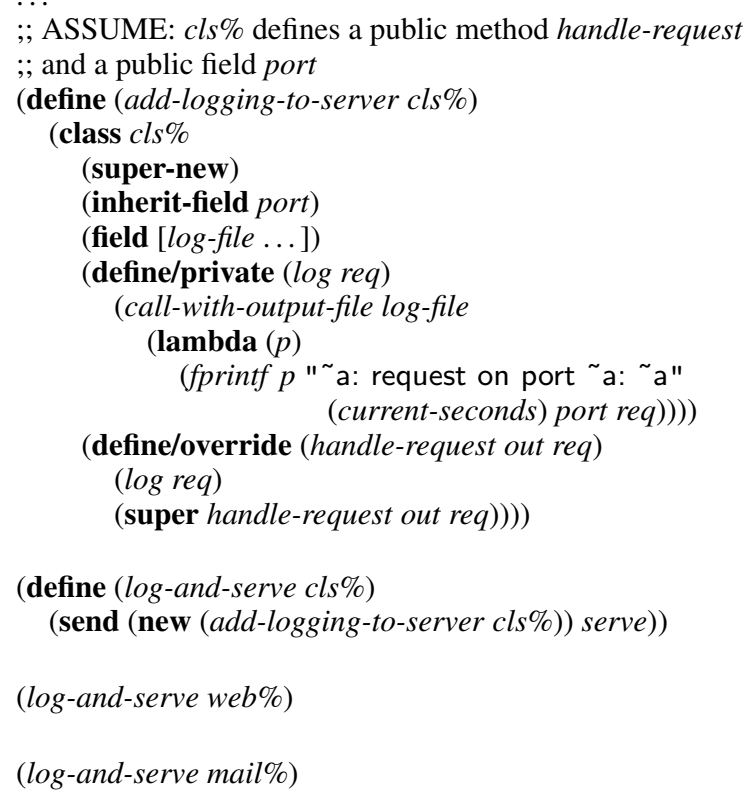

Figure 2. Using classes as class values

Figure 2 presents an example of using classes as first-class values. The first function, add-logging-to-server, subclasses its input class, overriding its handle-request method to add logging just as with logged-web\%. The second takes a class, adds logging via the first function, and then invokes the serve method on a new object of the resulting class. We then use the latter twice with the web\% class shown earlier and a class mail\%, a mail server with an interface similar to $w e b \%$ whose code is omitted.

The function add-logging-to-server, which takes a class that implements the handle-request method and extends it with logging behavior, is an example of a mixin. Bracha and Cook [7] were the first to propose these kinds of mixins, which provide an abstraction for extending classes with the same new behavior which is especially useful in a single inheritance language. As seen here, mixins are naturally expressed in a language with first-class classes.

\section{Contracts}

Behavioral software contracts provide a notation for describing the expected behavior and use of values via obligations and promises. We start with a description of the contract system of Eiffel [36], with which Meyer first popularized the idea of design by contract in the object-oriented world. We then switch to the contract system in PLT Scheme and elaborate on those attributes that shape our design of contracts for first-class classes: contract boundaries, blame tracking, and contracts for higher-order values.

\subsection{Contracts in Eiffel}

Eiffel supports three ways of describing contracts on classes: method preconditions, method postconditions, and class invariants. Method preconditions specify the condition that must hold when a 
method is called and are preceded by the require keyword. Method postconditions specify the condition that must hold when a method returns and are preceded by the ensure keyword. Class invariants describe conditions that must hold on every method entry and exit and are written at the class top-level in a block that begins with the keyword invariant. All contract features contain a sequence of boolean expressions, each tagged with a label that is used when reporting contract violations.

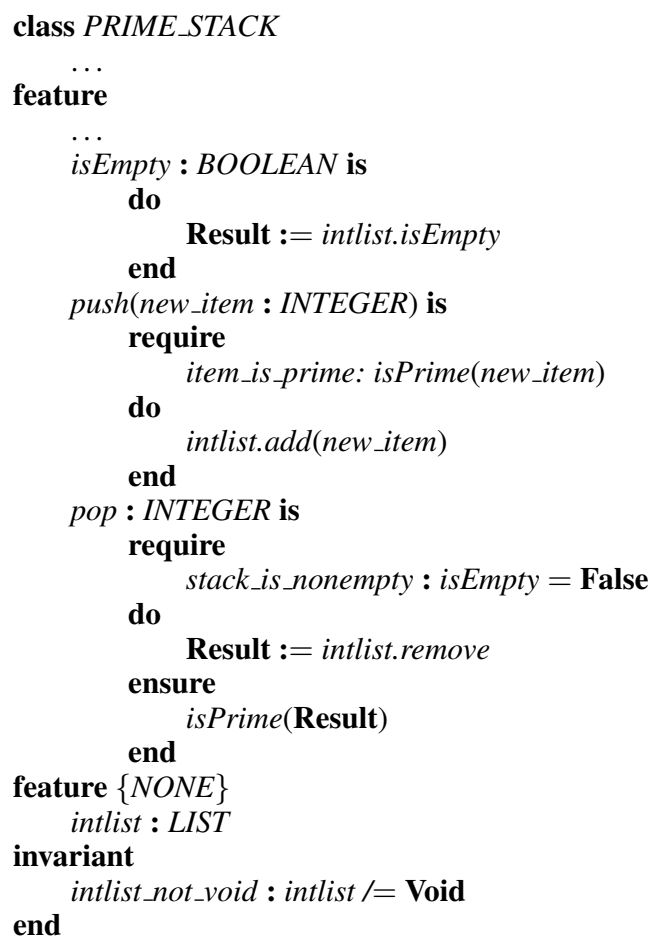

Figure 3. An Eiffel class for a stack of prime numbers

Figure 3 sketches a class that implements a stack of prime numbers. It assumes a predicate isPrime on integers and a class LIST that implements a mutable list with isEmpty, add and remove operations. Both add and remove are expected to operate on the same end of the list. It contains a private field intlist, in which the stack stores the items, and an invariant on intlist that requires it never be the value Void, which is the equivalent of Java's null. The class PRIME_STACK also provides three public operations: push, pop, and isEmpty. The push operation requires that its argument be a prime integer. The pop operation requires that intlist is not empty and guarantees that the result is a prime number.

Since all class invariants are checked on both method entry and exit, this can lead to a problem due to re-entrance [46, p.66]. Any method that needs to temporarily break an invariant must perform all the actions necessary to restore the invariant locally.

More recent object-oriented languages with contracts, such as Spec\# [4], provide the programmer with a mechanism to state that a block of code is allowed to break invariants. After the block is executed, the invariants are checked to ensure that they are properly re-established. PLT Scheme solves this problem in a different way.

\subsection{Contracts in PLT Scheme}

To illustrate contracts in PLT Scheme, figure 4 contains a module, written in the plain imperative fragment of the language with a "translation" of the Eiffel class plus two additional procedures. The contracts are written using a syntactic extension of PLT Scheme

$$
\text { \#lang scheme ；; name: prime-stack }
$$

(define intlist null)

(define (empty?) (null? intlist))

(define (push i) (set! intlist (cons i intlist)))

(define ( $p o p)$

(begin0 (car intlist)

(set! intlist (cdr intlist))))

(define (add-and-remove n) (push n) (pop))

(define (map-prime $f)$ (set! intlist (map f intlist)))

\section{(provide/contract}

[empty? $(. \rightarrow$. boolean?)]

[push (prime? . $\rightarrow$. void?)]

$\left[\right.$ pop $\left(()() \#\right.$ :pre (compose not empty?).$\rightarrow_{\mathrm{d}}$. prime?)]

[add-and-remove (number? . $\rightarrow$. number?)]

$[$ map-prime $(($ prime $? . \rightarrow$. prime? $) . \rightarrow$. void $?)])$

Figure 4. A PLT Scheme module for a stack of prime numbers

that allows prefix operators to be written in an infix manner. We use this extension to move $\rightarrow$ and $\rightarrow_{\mathrm{d}}$, the contract operators for procedures, between the domain and range contracts. The module, dubbed prime-stack, assumes the existence of a predicate on numbers called prime?. It provides push and pop operations on a stack of prime numbers. When a client imports the prime-stack module and uses this functionality, the argument to push and the result from pop are checked for primality. Expressions that are checked as preconditions can be specified in the $\rightarrow_{\mathbf{d}}$ contract form, as seen here in the contract of pop. Thus, we specify that the stack must be nonempty when pop is called.

\subsubsection{Contract Boundaries}

In contrast to Eiffel, contracts in PLT Scheme are checked only when values cross the module boundary. For our running example, a boundary crossing occurs when a value flows into or out of the prime-stack module. Calls internal to the module are not checked. Consider the nonsensical add-and-remove function. It first adds, then removes an arbitrary number from the stack. Since its calls to push and pop are internal to prime-stack, these calls are not checked and therefore do not signal a contract violation.

At first glance, contract checking at boundaries is too coarse grained to be useful. However, it eliminates the above-mentioned re-entrance problem, and it ensures that contract checking doesn't interfere with the implementation of tail calls, a critical element of proper object-oriented design. Furthermore, the experience of the PLT Scheme community pragmatically demonstrates that boundary-checked contracts are highly useful. Programmers tend to write small modules and trust the code within a single module. When contract violations are signaled, the resulting error limits the region of code that needs to be inspected. Programmers debug via unit tests and, if they fail to find the precise location of the error, employ define/contract and with-contract [45]. These create local contract boundaries that can narrow down the search in a module. 


\subsubsection{Blame Tracking}

Every module that imports prime-stack enters into an agreement with the prime-stack module. If a contract violation occurs, then the appropriate party to the contracts is blamed. For example, if a client calls the push function with a composite number, the contract monitoring system blames the client. If the client calls the pop function and a composite number were returned, a contract violation would be signaled that blames prime-stack.

When we discuss blame, we call the server the positive party and the client the negative party. These terms are analogous to the uses of the terms positive and negative to describe positions in implications in logic or in type systems.

\subsubsection{Contracts for Higher-Order Values}

Since PLT Scheme is a functional language, functions are firstclass values. Of course, it is impossible to check contracts, such as (prime? . $\rightarrow$.prime?), on functions at the point where the function crosses the contract boundary-instead, the contract system must delay checking until the function is applied. At application, the contract system can then check that the contracts on the arguments hold, and that the contracts on the return value(s) hold after the function is evaluated.

As seen with push and pop, the negative party is responsible for the contracts on the arguments to exported functions and the positive party is responsible for the contracts on values returned from exported functions. The reasoning behind this becomes clear when we consider functions as opening channels across the contract boundary, where arguments flow from the client to the server and then the result from the server to the client.

This reasoning generalizes to cases where the arguments provided by the client are functions, as in the argument $f$ to the primemap function. Then the arguments to those functions originate in the server, and thus the positive party is responsible for these arguments of arguments, while the negative party is responsible for the result. Indeed, the roles are swapped again if any of the doublynested arguments are functions. So higher-order function contracts require swapping the positive and negative parties each time we descend into the left-hand side of a function contract. For details, see Findler and Felleisen's paper [14].

\section{Adding Contracts to First-class Classes}

While contract systems have been around for object-oriented languages for a long time, none of them cope with first-class classes. The presence of first-class classes poses significant challenges to the introduction of contracts. In this section, we spell out the two linguistic problems; section 6 deals with the performance problem. First, a language with first-class classes must come with contracts that are separate from the classes that they protect. Second when entire classes flow across contract boundaries, the contract sublanguage must separate their roles as factories of new objects and as containers of reusable code.

See section 8 for the PLT Scheme-specific problem of mixing Beta-style inheritance into a conventional single-inheritance language. It poses additional linguistic problems.

\subsection{Separating Classes and Contracts}

In conventional object-oriented languages with contracts, say Eiffel or Spec\#, programmers write down contracts as part of a class or method. Writing down contracts next to fields and methods feels natural to programmers who know only fixed class hierarchies. In a language with first-class classes, however, contracts must come separate from classes. After all, classes flow across contract boundaries just like numbers, strings, or objects.
The flow of classes across contract boundaries can take all shapes and forms. Consider a class-consuming method (or function). Like its cousin that demands a prime number, this method should be able to require that its input - a class - meet certain conditions. Similarly, a class-producing method (or function) should be able to make promises about its results. Now if we think of mixins as "functions" that extend unknown superclasses, it becomes obvious that class contracts are the natural way to specify their promises and obligations. Similarly, trait manipulating operations that alias some methods, remove others, and modify a third class can be described with such class contracts, too.

\subsection{The Role of Classes}

Classes play two major roles in an object-oriented language: they serve as blueprints for the creation of new objects, and they serve as containers of code. While the former role is well-explored from a contract perspective, the latter role demands a new look because classes are no longer in a fixed relationship. Indeed, one and the same class may extend many different superclasses, even though the language supports only single inheritance. Consequently we must decide whether a subclass is required to be consistent with the superclass's behavior [30] or whether it exploits the superclass solely as a code repository.

As far as contracts are concerned, the separate roles of classes pose two design problems. First, we need to decide whether a class contract should specify both roles jointly or whether a contract may specify the two different aspects separately. Second, we must also decide whether contracts protect implementations or interfaces.

For guidance, we inspected the PLT Scheme code base (close to $500 \mathrm{Kloc}$ ). This inspection suggests that the creators of classes wish to impose different obligations and expect different promises from subclasses and from objects. Our contracts therefore allow the specification of obligations on subclasses, similar to but also different from so-called specification interfaces [25]. Furthermore, the code inspection also implies that many uses of class hierarchies are about implementation inheritance rather than type inheritance. ${ }^{3}$ The design of our class contracts therefore focuses on protecting implementations, not behavioral type relationships.

\section{A Tour of Contracts for First-class Classes}

The main construct of our class contracts is a contract combinator that combines clauses. Each clause is an assertion on visible fields and methods. Following the analysis of the preceding section, the clauses come in two flavors: for external method calls and for the specification interface. The latter are clauses that govern the flow of values between methods in superclasses and subclasses.

\subsection{A Contract Combinator for Class Values}

Class contracts are specified with the class/c combinator. Like the contract combinator for first-class functions, class/c exhibits higher-order flavor. After all, a class denotes a collection of objects, i.e., values with behavior, and it is impossible to check behavioral aspects all at once. For example, contracts for methods are checked like contracts for functions-one application at a time. Contracts on initialization arguments provide a second example, as contract application must be delayed until the actual instantiation is performed. Similarly, contracts on fields cannot be checked before an object is created. Hence, our contract system applies the contract to the value in a field whenever the field is accessed or mutated from across a contract boundary.

Figure 5 demonstrates the PLT Scheme equivalent of the PRIME_STACK class from figure 3. In the class/c contract, each

\footnotetext{
${ }^{3}$ It is possible and indeed likely that PLT Scheme's untyped nature biases our results here and that a typed language has different needs.
} 


\subsection{Contracts and Objects}

While a class contract specifies the behavior of every object of a protected class, in some cases a programmer may wish to place a special contract on some object. One abstract example is the Singleton pattern [18]. Since only one instance is provided to clients, the class itself never crosses the contract boundary. Hence, we must attach a contract to the object as it flows out of the module.

Since an object has already been fully initialized and it doesn't play the role of a code container in a class-based language, only external field and method clauses appear in object contracts. Also, the uncontracted and contracted versions of the object share the same state. If the client retrieves the value from a field that is inconsistent with that field's contract, then the server is blamed.

A programmer may also wish to enforce a stronger contract on a particular object than on its class. Take the prime stack implementation in figure 5. In the same module, we may wish to create an object from that class and ensure that it is used only with prime numbers whose value is greater than $1,000,000$. Figure 8 contains an appropriate code snippet.

(define (big-prime? n) (and (prime? n) (>n 1000000)))

(define big-prime-stack (new pstack\%))

(provide/contract

[big-prime-stack

(object/c [empty? (any/c. $\rightarrow$. boolean?)]

[push (any/c big-prime? . $\rightarrow$. void?)]

[pop (non-empty-stack? . $\rightarrow$. big-prime?)])])

Figure 8. A prime stack object with a stronger contract

\subsection{Contracts and Class Inspection}

PLT Scheme, like other object-oriented languages, comes with operations for inspecting the relationships between objects and classes and among classes. Specifically, is-a? determines whether some object is an instance of some class, and subclass? checks whether a given class is a subclass of another one. This, in turn, raises the question whether an object or class extension satisfies the same relationships with a contracted version of the class.

Figure 9 contains a program with multiple contract boundaries; a pictorial view of the same program is given in figure 10. In the latter, vertical lines represent contract boundaries, squares are classes, and circles denote instances. The program contains three modules, and each exports an object and a single contracted class. Here, we must decide whether the objects that were created using the uncontracted versions of the exported classes still have an is-a? relationship to the contracted versions.

For our design decision, we accept the principle that the removal of contracts should not affect the observable outcome if the program with contracts evaluates to a plain value. The theory of contracts as projections [12] explains this principle from a theoretical perspective. A projection is roughly speaking a function that maps values to values with "less" behavior or, more appropriately here, values where some part of the behavior has been replaced by errors. In the case of contract projections, the act of applying a projection turns inappropriate behavior into an error signal that represents a contract violation.

When we apply this principle to the is-a? and subclass? checks, the consistent answer is that a class with a contract should act as much as possible as its counterpart without contracts. Since, in the absence of contracts, is-a? and subclass? would return true for objects and subclasses of a given class, then they must also return

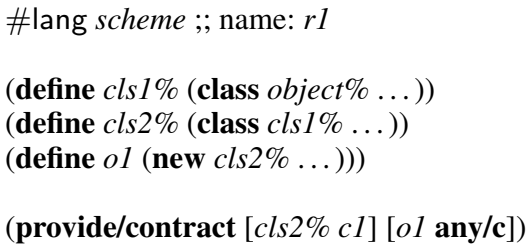

\begin{tabular}{|c|c|}
\hline $\begin{array}{l}\text { \#lang scheme ; name: } r 2 \\
\text { (require } r 1 \text { ) } \\
\text { (is-a? ol cls } 2 \% \text { ) }\end{array}$ & $\begin{array}{l}\text { \#lang scheme ; name: } r 3 \\
\text { (require } r 2 \text { ) }\end{array}$ \\
\hline $\begin{array}{l}(\text { define } o 2(\text { new } c l s 2 \% \ldots)) \\
(\text { define } c l s 3 \% \\
\quad(\text { class } c l s 2 \% \ldots)) \\
(\text { define } o 3(\text { new } c l s 3 \% \ldots)))\end{array}$ & $\begin{array}{l}\text { (is-a? } o 3 \text { cls3\%) } \\
\text { (define } 04(\text { new } c l s 3 \%)\end{array}$ \\
\hline $\begin{array}{l}\text { (provide/contract } \\
{[\text { cls3\% c2] }[03 \text { any/c] })}\end{array}$ & \\
\hline
\end{tabular}

Figure 9. Example uses of is-a?

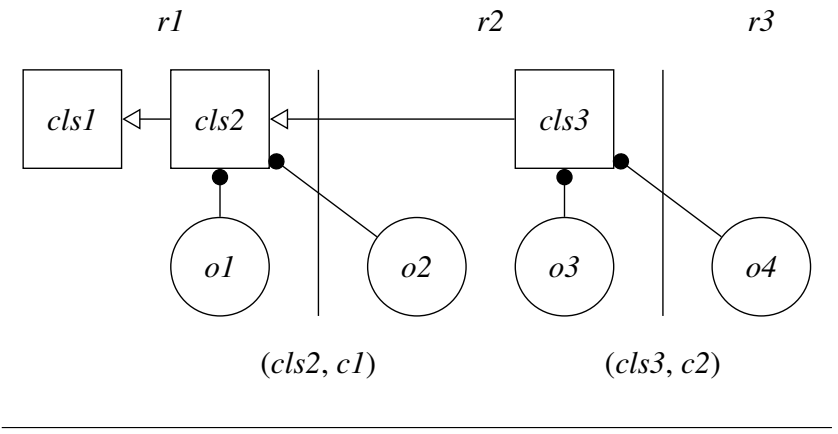

Figure 10. Classes, objects, and contract boundaries

true when those same objects and subclasses are compared against the contracted version of the class. Thus, all the queries in figure 9 return true, since they would do so in a version of the program where none of the class definitions were contracted.

\section{Implementation}

The Findler-Blume explanation of contracts as pairs of mathematical projections also motivates an implementation of a contract monitoring system. For a language that uniformly views values as firstclass entities, the implementation of a contract is almost a transliteration of the mathematical model into functional code.

Thus, class/c denotes a function that maps the (names of the) two contract parties into a pair of projections from classes to classes. One way to understand this idea is to compare figures 10 and 11 . In the latter figure, the dotted squares represent classes created from applying projections to classes. The inheritance arrows connect the derived classes to the originals. Of course, a complete understanding of the process requires some background concerning the implementation of classes and objects in PLT Scheme. 


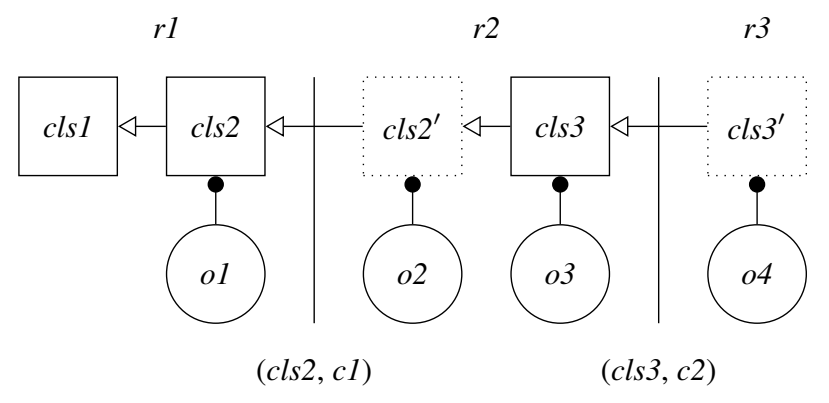

Figure 11. New classes resulting from contract application

\subsection{Basics}

Both classes and objects are implemented as heterogeneous and opaque structures. ${ }^{4}$ Opaqueness guarantees safety. The structure contains all the necessary information for constructing objects and looking up methods and fields. Object construction is handled by functions that create structural representations of objects using the initialization arguments and an auxiliary function formed from the initialization expressions of the class (see section 2.1).
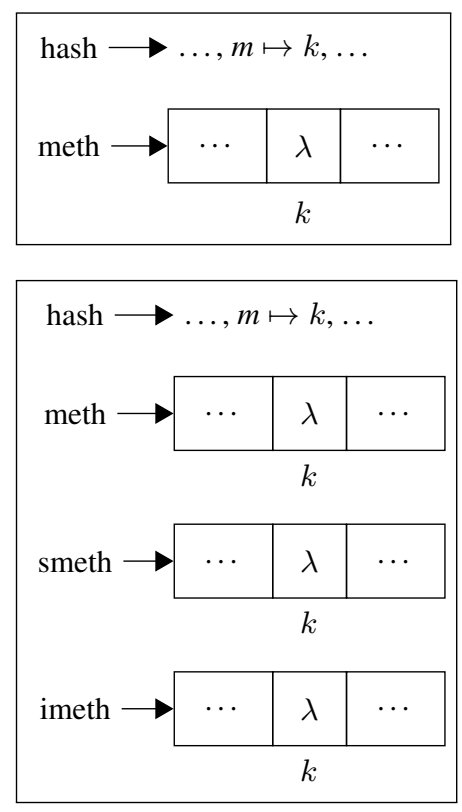

Figure 12. Method table organization, original and first revision

As usual, the class representation contains a vector of methods and a hash table that maps a method name into an index for the vector. Fields are represented in the same fashion, except that instantiated objects contain the vector of field values. The class compiler transforms both a field access and a method call into computations that use the appropriate vector slot. The compiler can compute the vector index for internal uses of fields and methods statically, whereas the index must be computed at runtime for external references. Field assignments are converted into vector assignments. Calls to super methods are compiled via indexing computations into the method table of the super class.

\footnotetext{
${ }^{4}$ PLT Scheme's class system is technically implemented via a macro library, though it is programmatically indistinguishable from a native class system.
}

In short, all method calls access the same method vector, whether they are internal calls, external sends, or super calls in a subclass. Each kind of contract clause affects a different set of uses, however. We could store the different projections and apply the correct projection for a particular use, but applying contract projections can be a costly operation. Our implementation therefore trades space in the class representation for run-time speed; that is, it separates out the method vector into three vectors, illustrated by figure 12. External calls via send use the first vector (meth), super calls the second (smeth), and direct internal calls the third (imeth). In support, we change the compiler to convert each operation into an indexed access into the appropriate vector. This separation immediately supports eager projection application to the methods accessed by calls via send and super calls; we describe the additional changes required for internal calls in section 6.2.

For mutable fields, we cannot apply contract projections upon object creation. Since the value of a field changes over time, we apply appropriate projections whenever a field is accessed or mutated across a contract boundary. To this end, our compiler stores two pairs of functions for each field. One pair protects external uses, and the other pair protects internal uses. Each pair contains an accessor and a mutator function. When a contract with a field-related clause is applied to a class, the appropriate pair of functions is replaced with a new pair. The new accessor function applies the appropriate projection with covariant blame to the result of the old accessor. The new mutator function applies the projection with contravariant blame to the incoming value and then calls the old mutator.

To protect initialization arguments, the compiler creates a contracted initialization function. It consumes the same arguments as the original, applies the appropriate projection to each argument, and passes the contracted arguments to the original initialization function. The only trickiness is due to keyword initialization of arguments, where multiple classes in a single hierarchy can have initialization arguments of the same name. These overlapping names are resolved in order from subclass to superclass, so we must consider the order in which contracts are specified. Of course, a Javalike initialization method does not pose this problem.

\subsection{Internal Dynamic Dispatch}

The revised object organization as it stands cannot support contracts on dynamic dispatches within the class hierarchy. Such method calls should be affected only by contract boundaries between the class with the call and the class that contains the method definition. We therefore employ a vector for internal calls where each entry maps to a vector of method implementations. Also, we need to ensure that override contracts are not lost when a method is overridden, so each class also contains an analogous table of vectors of projections. The separation of vector entries based on contract boundaries means that contract projections for internal dynamic dispatch can be eagerly applied when creating the contracted class value, and that we can apply different contracts on different sides of the boundary. The resulting changes to the class structure are illustrated in figure 13 .

Figure 14 contains an example class hierarchy where one of the classes, $c l s 2$, is contracted with inherit and override clauses for the method $m$. Figure 15 depicts the method and projection vectors used for internal calls to $m$ in each class. Class $c l s 2$ is shown twice: $c l s 2$ denotes the class without contracts, and $c l s 2^{\prime}$ depicts the class with contracts applied. The different method implementations are differentiated using subscripts, and primed method names denote application of contract projections to methods.

The vectors for a given method in a class contains an entry for each contract boundary between that class and the first class that defined the public method in the class hierarchy. In our example, $c l s 1$ and $c l s 2$ both use a one-element vector for method $m$ since 


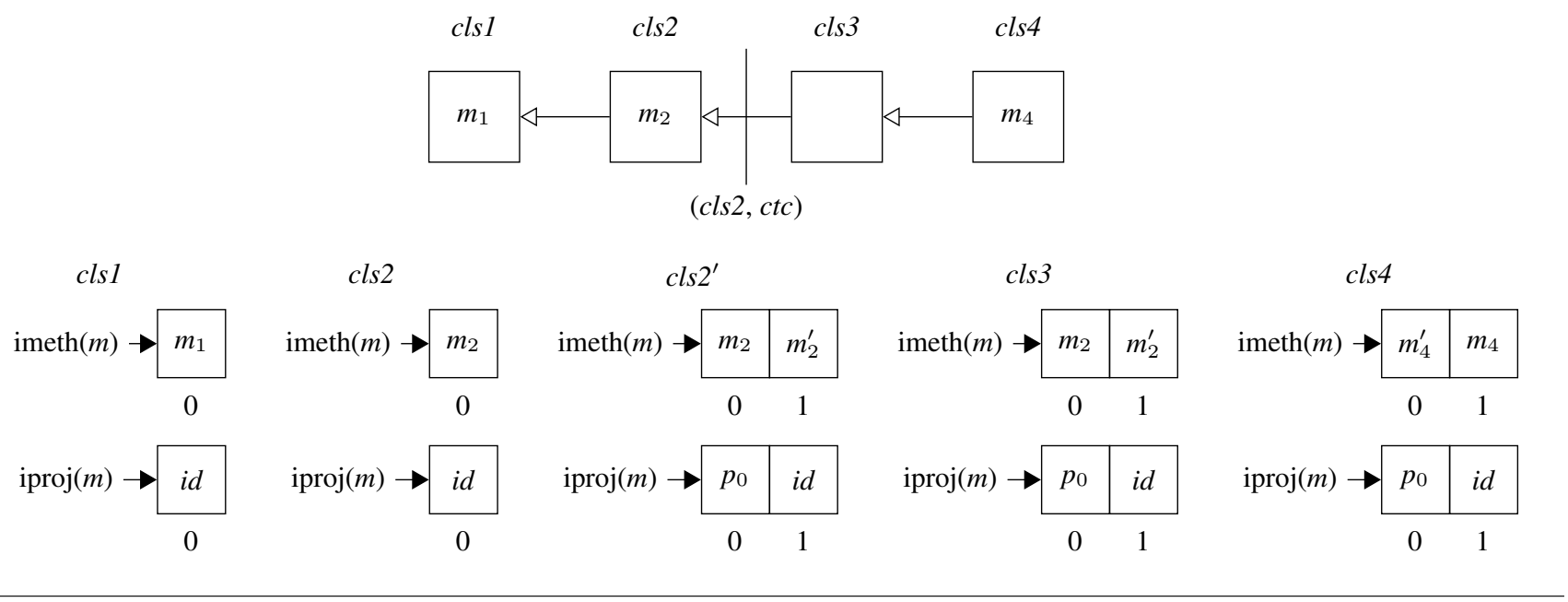

Figure 15. Dispatch tables for figure 14

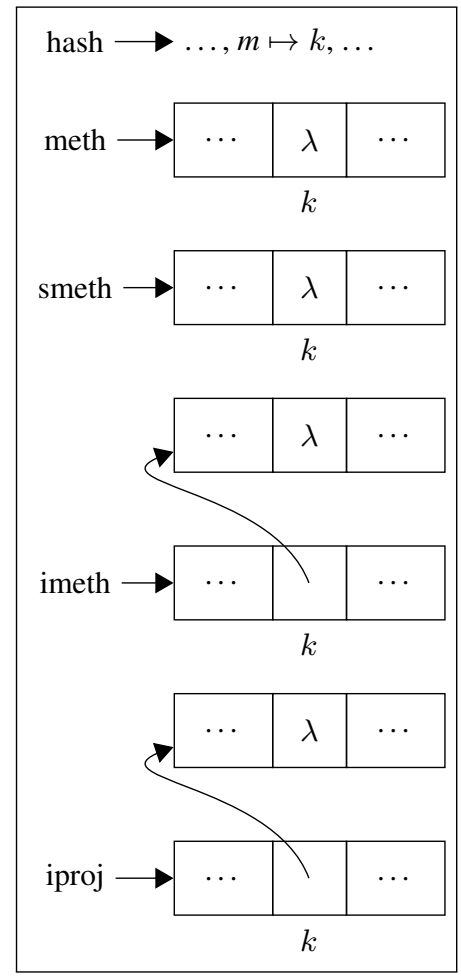

Figure 13. Second revision of class method tables

there are no contract boundaries between them and $c l s l$, the first class to define $m$. In contrast, the contract region introduced by provide/contract separates $c l s 3$ and $c l s 4$ from $c l s 2$, meaning the two classes use a vector of two elements for method $m$.

The compiler turns an internal method call into an access into the internal method table of this where the secondary index takes into account the number of contract boundaries between the current class and the first class to define the targeted method. Consider an instance of $c l s 4$. If code using that instance calls $n$, the call from cls 1 crosses the contract boundary to use the overridden method in cls4. Since the call in $c l s 1$ is compiled to retrieve the method from

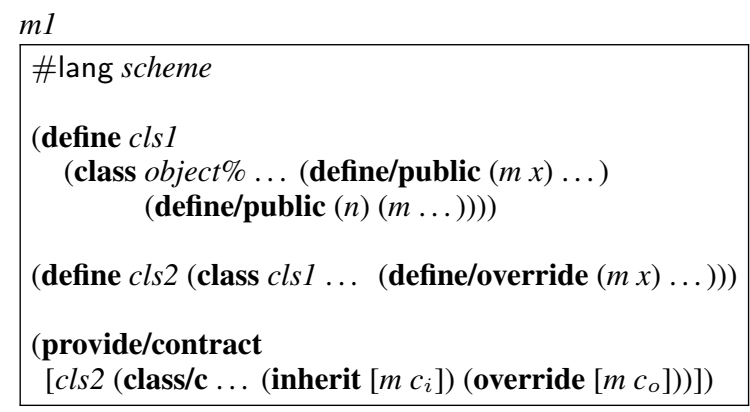

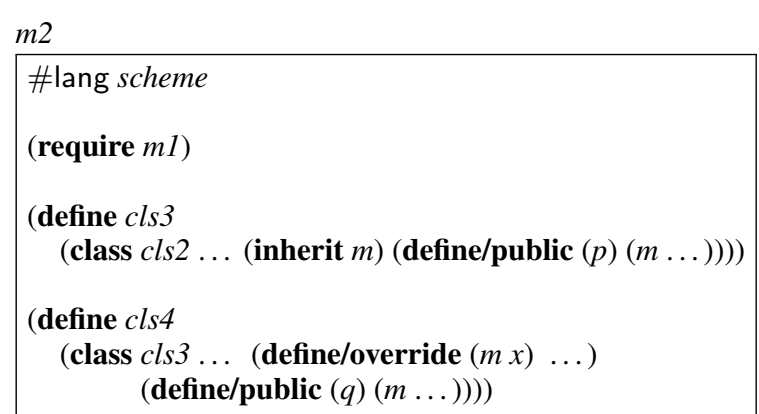

Figure 14. Example classes with override and inherit contracts

index 0 , it uses the contracted method $m_{4}^{\prime}$. If the code calls $q$, the internal method call from $\mathrm{cls} 4$ uses index 1 . Thus, it retrieves $m_{4}$, i.e., the uncontracted method.

If a class includes a new public method, the compiler creates two new vectors with one slot. The method vector contains the method implementation, and the projection vector contains the identity projection. See the diagram for method $m$ in $c l s 1$.

If a subclass does not override a method, as in $c l s 3$, its method and projection vectors are the same as its superclass. If it does override the method, as in $c l s 2$ and $c l s 4$, then the projection vector remains the same but the method vector is the result of applying the new method implementation to each projection from the corresponding entry in the projection vector. In $c l s 4$, the method at index 1 is $m_{4}^{\prime}$, the result of composing $m_{4}$ with the projection $p_{o}$. The method at index 1 is just $m_{4}$, since index 1 of the projection vector still contains the identity projection. 
The application of a class contract to a class yields a new class that contains extended method and projection vectors. In particular, an inherit clause for a method means that the compiler wraps high-indexed entries in the method vector with appropriate contract checks (until that method is overridden). Similarly, an override clause means the compiler stores the appropriate projection in lowindexed entries of the projection vector, so that they are available for creating the method vector for an overridding subclass.

The creation of the method and projection vectors for $c l s 2^{\prime}$ from those in $c l s 2$ follows these rules. The method in index 0 has been copied, while the method $m_{2}^{\prime}$ at the new index 1 is the result of applying the contract projection for $c_{i}$ (from the class contract for cl2) to $m_{2}$. The projection in index 0 is the result of composing the new projection for $c_{o}$ to the identity projection, and the projection at the new index 1 is the identity projection.

\subsection{Object Contracts}

Since classes in PLT Scheme are values, the contract combinator for objects can be described in terms of the contract combinator for classes. Operating on an object with a contract—where the contract specifies the behavior of public methods and fields on one particular object-is equivalent to operating on an object that shares the same state but is based on a class equipped with the appropriate contracts.

When an object flows across a contract boundary with an object contract, our run-time system creates a new object whose methods are proxies for the original methods and whose field selectors and mutators forward actions to the original object. This forwarding guarantees that the two objects - the original and the one with the object contract-share state as desired. Figure 16 explains the idea pictorially, using a diamond on the right-hand side of the contract boundary to represent a proxied object. The diagram also points out that this latter object contains a reference to the contracted class for method access and a reference to the original object for fields.

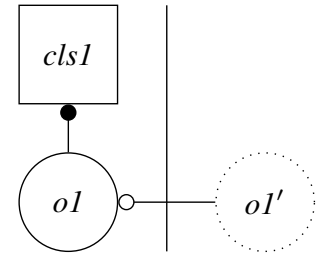

$(o 1, c)$

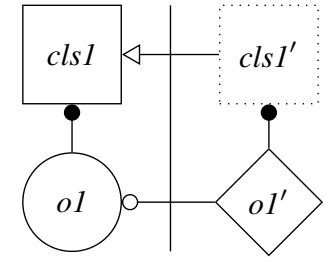

$(c l s 1, c)$
Figure 16. Example application of an object contract

To ensure that is-a? and subclass? return the same result on wrapped classes and/or objects, the class system must determine that two classes are equal modulo contract wrapping. For this, each class contains a reference to the uncontracted version of the class. In classes that have not been wrapped, this is a self-reference. Otherwise, the new contracted class copies the reference from the original class during contract application. Thus, the checks in the implementations of is-a? and subclass? now use reference equality on the uncontracted class.

\section{Evaluation}

Our implementation strategy affects the performance of all operations on both classes and objects. Worse, even classes and objects that do not use contracts pay a price. The latter effect is mostly noticeable in the context of micro-benchmarks that stress-test various features of the class system. Our micro-benchmarks compare three versions of the PLT Scheme code base: the initial code base, dubbed "pre;" the "class/c" code base, which runs all existing code using the new class compiler; and the "object/c" code base, in which object contracts are implemented via the revised class compiler. Since none of the benchmarks use class contracts, the difference in running times describe precisely the added overhead of the new implementations.

The results of our micro-benchmarks are collected in figure 17. All times are given in milliseconds, and for each test we provide the average running time, the minimum running time, the maximum running time, and the average running time normalized to the "pre" version. We also list how often the benchmark executes the measured feature. Notice that the local and inherited field benchmarks contain an order of magnitude more trials than the first three, and the instantiation and inheritance tests an order of magnitude fewer. The four benchmarks for subclass creation differ in whether new fields and/or methods are introduced.

When only class contracts are introduced, the overhead on even these class-focused benchmarks is quite small-usually less than $5 \%$. In a couple of cases, the average running time actually drops because our revised class compiler can better optimize field accesses due to the separation of internal and external accesses. Once object contracts and thus object proxying are added, however, the overhead increases noticeably in all cases but external accesses.

For internal field access - a fairly common use case-the impact of proxying is huge. While the original compiler translates field references into direct structure dereferences, the revised compiler must take into account that some objects are proxy objects. Hence all internal field references have slowed down by ca. $350 \% .^{5}$

Fortunately micro-benchmarks tend to exaggerate the effect of these compiler changes. In a realistic code base, internal field references aren't used for every other instruction, and methods perform real work rather than return the same numeric value for every call. One such large code base in our possession is DrScheme [13], a standard "macro" benchmark for our team (PLT). DrScheme is a Scheme-specific graphical IDE whose implementation makes heavy use of classes and objects. The team keeps track of the runtime for an extensive automated interaction test of the IDE. Figure 17 also contains the results for these automated interaction test on the three revisions. Our measurements for adding class contracts shows no noticeable impact. Indeed, even the overhead of object proxying is a tolerable $5 \%$.

In summary, the addition of contracts to our language of firstclass classes has some impact on all class-based programs, not just those that employ contracts. While micro-benchmarks suggest that this cost is significant for some features of the class system, the load for a complex system (approx. 250,000 lines of code) is almost too small to be noticed.

\section{Adding Beta-style Augmentation}

PLT Scheme supports not only conventional method overriding but also Beta-style method augmentation [20]. Indeed programmers routinely mix and match the two styles. Here we explain how these extensions interact with contracts.

\subsection{Mixing Inheritance Styles}

In conventional class-based languages, subclasses may replace methods with overriding definitions. In contrast, Beta [28] and gbeta [10] demand that subclasses provide method augmentations, which the super method may utilize. This shift of perspective favors behavioral subtyping; after all, the superclass dictates the primary behavior of a method and where it may change.

Figure 18 shows how PLT Scheme supports Beta-style inheritance for the example from figure 1. The handle-request method

\footnotetext{
${ }^{5}$ We are currently investigating an alternate implementation strategy for objects, which should eliminate a good portion of this overhead.
} 


\subsection{Contracts for Beta-style Inheritance}

Since conventional and Beta-style inheritance complement each other, we need to add clauses for Beta-style inheritance to class/c. Consider figure 20, which provides an example of a class hierarchy that crosses a contract boundary and utilizes both augmentable and overrideable methods. One clear requirement is to declare the behavior expected from augmenting methods, so that the contract system can protect uses of inner. To this end, we add another type of clause to class/c, similar to that for super, but with the keyword inner. Since this specification covers a form inside the contract boundary that depends on a value from a subclass outside, blame is handled in a contravariant manner. The default expression, which lies within the contract region, is not checked.

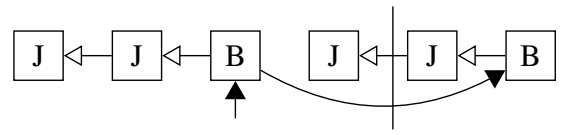

Figure 20. A mixed inheritance chain and a contract boundary

In addition to the inner form, we add two new forms, augment and augride. These forms perform double duty: they describe the obligations to subclasses for internal uses of the listed functions, on which we later elaborate, but they also document what style of inheritance is allowed for extending the method in subclasses.

- augment clauses list methods that must be augmented, and

- augride clauses list methods that must be overridden.

Figure 21 shows a mixin similar to that from figure 7, but using Beta-style inheritance like the examples in figure 18. Through its input contract, it places a requirement on how the superclass can use handle-request through inner, and likewise describes how it expects future subclasses to further augment handle-request.

\subsection{Implementation}

To implement this new behavior for inner contracts, the class structure contains a projection for each augmentable method which defaults to the identity projection. Every time a class/c projection is applied, any inner contracts are converted to projections and composed with the current projection. When a subclass augments the method in question, the projection is applied to the augmenting definition when it is inserted into the inner method table. If the augmenting definition is overrideable, then any overriding definition also gets the projection applied before it replaces the original augmentation. Once the method again becomes augmentable, the projection is reset to the identity projection.

The augride and augment clauses describe methods that have been previously augmentable in the class hierarchy. For such methods, the first augmentable definition will always be the target of dynamic dispatch as illustrated in figure 19. Thus, instead of protecting uses of the method in the contracted class from overriding implementations like override, these contracts protect the first augmentable definition in the contracted class from uses in subclasses.

This change in behavior requires a small change to our implementation strategy for internal dynamic dispatch. The compiler handles methods protected by override contracts as outlined in the previous section. For a augment or augride clause, which can be used only if the corresponding method has been augmentable at least once, we change our strategy. The class/c projection does not store the augment and augride projections in the projection vector; instead, the class/c projection applies these projections like those for inherit. Since a once-augmentable method cannot be overridden as a target of dynamic dispatch, these contracts will be continue to be checked for all subclasses.

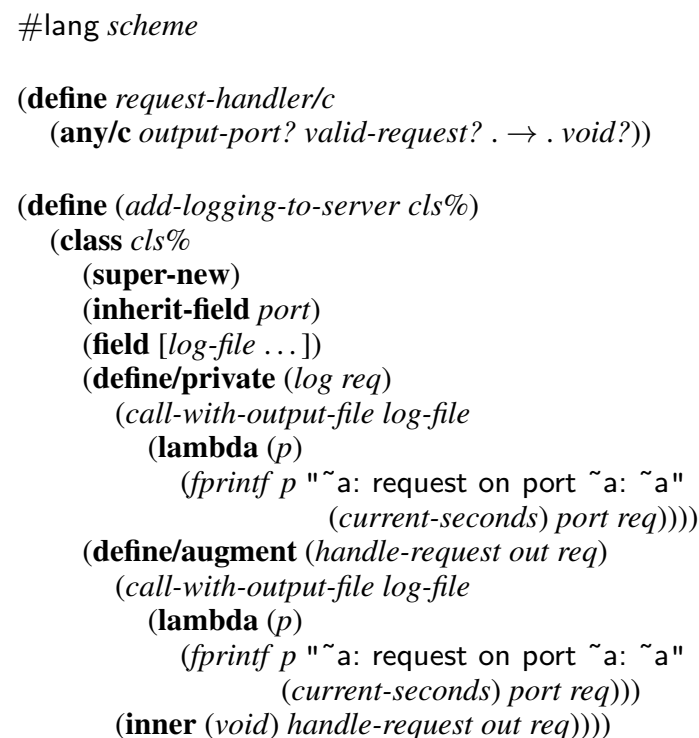

(provide/contract

[add-logging-to-server

$(\rightarrow$ (class/c (inherit-field [port tcp-port?])

(inner [handle-request request-handler/c]))

(class/c (field [log-file valid-path?])

(inner [handle-request request-handler/c])

[handle-request request-handler/c]))])

Figure 21. A contract for an augmenting mixin

\section{Related and Future Work}

While Parnas [39] introduced the idea of using declarative specifications of expected behavior between different software components, Meyer [34, 35] named them contracts and, with his design of Eiffel [36], put them on the map for software engineers and language designers. Later work by Carrillo-Castellon et al. [8] and Plösch [40] added similar contract systems to dynamically typed object-oriented languages. Findler and Felleisen [11] revisited Meyer's contracts with a focus on blame tracking. Contracts for higher-order functions are also due to Findler and Felleisen [14].

The contracts presented in this paper provide a solid basis for protecting classes in a fashion consistent with implementation inheritance. However, there are two extensions to this work that we wish to tackle in the future: interface inheritance and declaration of contracts inside class definitions.

First, PLT Scheme, like other object-oriented languages, enables the programmer to specify interfaces and attach them to classes. These interfaces describe sets of public methods that a class must implement. Class definitions can then list sets of implemented interfaces. Our plan calls for interfaces with contracts. Thus, classes that implement a contracted interface will have their methods checked according to behavioral subtyping [11].

Second, we would like to provide the programmer with the ability to declare contracts on class features inside a class definition, instead of contracting the resulting value after the fact. However, as in our previous work on contracts for first-class modules [44], this means that the class itself is a contract boundary. Doing so requires further research into how class-level contract boundaries interact with each other and other contract boundaries. 


\section{References}

[1] Eric Allen, Jonathan Bannet, and Robert Cartwright. A first-class approach to genericity. In Object-Oriented Programming, Systems, Languages, and Applications, pages 96-114, 2003.

[2] Eric Allen, David Chase, Joe Hallett, Victor Luchangco, Jan-Willem Maessen, Sukyoung Ryu, Guy L. Steele Jr., and Sam Tobin-Hochstadt. The Fortress Language Specification. Sun Microsystems, 2008.

[3] Davide Ancona, Giovanni Lagorio, and Elena Zucca. Jam-designing a Java extension with mixins. ACM Trans. Program. Lang. Syst., 25(5):641-712, 2003.

[4] Mike Barnett, K. Rustan, M. Leino, and Wolfram Schulte. The Spec\# programming system: An overview. In CASSIS 2004, volume 3362 of LNCS, pages 49-69. Springer, 2004.

[5] Detlef Bartetzko, Clemens Fischer, Michael Moller, and Heike Wehrheim. Jass - Java with assertions. Electronic Notes in Theoretical Computer Science, 55(2):103-117, 2001.

[6] Antoine Beugnard, Jean-Marc Jézéquel, Noël Plouzeau, and Damien Watkins. Making components contract aware. In IEEE Software, pages 38-45, June 1999.

[7] Gilad Bracha and William Cook. Mixin-based inheritance. In Object-Oriented Programming, Systems, Languages, and Applications/European Conference on Object-Oriented Programming, pages 303-311, Ottawa, Canada, 1990.

[8] M. Carrillo-Castellon, J. Garcia-Molina, E. Pimentel, and I. Repiso. Design by contract in Smalltalk. Journal of Object-Oriented Programming, 7(9):23-28, 1996.

[9] Dominic Duggan and Ching-Ching Techaubol. Modular mixin-based inheritance for application frameworks. In Object-Oriented Programming, Systems, Languages, and Applications, pages 223-240, 2001.

[10] Erik Ernst. gbeta-a Language with Virtual Attributes, Block Structure, and Propagating, Dynamic Inheritance. $\mathrm{PhD}$ thesis, University of Aarhus, Århus, Denmark, 1999.

[11] R. Findler and M. Felleisen. Contract soundness for object-oriented languages. In Object-Oriented Programming, Systems, Languages, and Applications, pages 1-15, 2001.

[12] Robert Bruce Findler and Matthias Blume. Contracts as pairs of projections. In Functional and Logic Programming, volume 3945 of LNCS, pages 226-241. Springer, April 2006.

[13] Robert Bruce Findler, John Clements, Cormac Flanagan, Matthew Flatt, Shriram Krishnamurthi, Paul Steckler, and Matthias Felleisen. DrScheme: A programming environment for Scheme. Journal of Functional Programming, 12(2):159-182, March 2002.

[14] Robert Bruce Findler and Matthias Felleisen. Contracts for higherorder functions. In International Conference on Functional Programming, pages 48-59, October 2002.

[15] Matthew Flatt et al. PLT Scheme. Reference Manual PLTTR2009-reference-v4.2.1, PLT Scheme Inc., January 2009. http: //plt-scheme.org/techreports/.

[16] Matthew Flatt, Robert Bruce Findler, and Matthias Felleisen. Scheme with classes, mixins, and traits. In Asian Symposium on Programming Languages and Systems, pages 270-289, November 2006.

[17] Matthew Flatt, Shriram Krishnamurthi, and Matthias Felleisen. Classes and mixins. In Symposium on Principles of Programming Languages, pages 171-183, January 1998.

[18] Erich Gamma, Richard Helm, Ralph Johnson, and John Vlissides. De sign Patterns: Elements of Reusable Object-Oriented Software. Addison Wesley, Massachusetts, 1994.

[19] Adele Goldberg and David Robinson. Smalltalk-80: The Language and its Implementation. Addison-Wesley, 1983.

[20] David S. Goldberg, Robert Bruce Findler, and Matthew Flatt. Super and inner - together at last! In Object-Oriented Programming, Systems, Languages, and Applications, pages 116-129, 2004.

[21] Benedict Gomes, David Stoutamire, Boris Vaysman, and Holger Klawitter. A Language Manual for Sather 1.1, August 1996.
[22] Murat Karaorman, Urs Hölzle, and John Bruno. jContractor: A reflective Java library to support design by contract. In Proceedings of Meta-Level Architectures and Reflection, volume 1616 of Lecture Notes in Computer Science, pages 175-196, July 1999.

[23] Michael Kölling and John Rosenberg. Blue: Language Specification, version $0.94,1997$.

[24] Reto Kramer. iContract: The Java design by contract tool. In Technology of Object-Oriented Languages and Systems, page 295, 1998.

[25] John Lamping. Typing the specialization interface. SIGPLAN Not., 28(10):201-214, 1993.

[26] B. W. Lampson, J. J. Horning, R. L. London, J. G. Mitchell, and G. J. Popek. Report on the programming language Euclid. ACM Sigplan Notices, 12(2), February 1977.

[27] Gary T. Leavens, Yoonsik Cheon, Curtis Clifton, Clyde Ruby, and David R. Cok. How the design of JML accommodates both runtime assertion checking and formal verification. Sci. Comput. Program., 55(1-3):185-208, 2005.

[28] Ole Lehrmann Madsen, Birger Møller-Pedersen, and Kristen Nygaard Object-oriented programming in the BETA programming language. Addison-Wesley Publishing Co., New York, NY, USA, 1993.

[29] Xavier Leroy. The Objective Caml system, Documentation and User's guide, 1997.

[30] Barbara H. Liskov and Jeannette M. Wing. A behavioral notion of subtyping. ACM Transactions on Programming Languages and Systems, 16(6):1811-1841, November 1994.

[31] D. C. Luckham and F.W. von Henke. An overview of Anna, a specification language for Ada. In IEEE Software, volume 2, pages 9-23, March 1985.

[32] Man Machine Systems. Design by contract for Java using JMSAssert. http://www.mmsindia.com/DBCForJava.html, 2000.

[33] Sean McDirmid, Matthew Flatt, and Wilson Hsieh. Jiazzi: new-age components for old-fashioned Java. In Object-Oriented Programming, Systems, Languages, and Applications, pages 211-222, 2001.

[34] Bertrand Meyer. Object-oriented Software Construction. Prentice Hall, 1988.

[35] Bertrand Meyer. Applying design by contract. IEEE Computer, 25(10):40-51, October 1992.

[36] Bertrand Meyer. Eiffel: The Language. Prentice Hall, 1992.

[37] David A. Moon. Object-oriented programming with flavors. In Object-Oriented Programming, Systems, Languages, and Applications, pages 1-8, 1986.

[38] Martin Odersky. The Scala Language Specification. Ecole Polytechnique Fédérale de Lausanne, 2009.

[39] David L. Parnas. On the criteria to be used in decomposing systems into modules. Communications of the ACM, 15:1053-1058, 1972.

[40] R. Plösch. Design by contract for Python. In IEEE Proceedings of the Joint Asia Pacific Software Engineering Conference, page 213, 1997.

[41] Didier Rémy and Jérôme Vouillon. Objective ML: A simple objectoriented extension of ML. In Symposium on Principles of Programming Languages, pages 40-53, January 1997.

[42] Nathanael Schärli, Stéphane Ducasse, Oscar Nierstrasz, and Andrew Black. Traits: Composable units of behavior. In European Conference on Object-Oriented Programming, volume 2743 of LNCS, pages 248274. Springer, July 2003.

[43] Michael Sperber, R. Kent Dybvig, Matthew Flatt, Anton Van Straaten, Robby Findler, and Jacob Matthews. Revised ${ }^{6}$ report on the algorithmic language Scheme. Journal of Functional Programming, 19(Supplement S1):1-301, 2009.

[44] T. Stephen Strickland and Matthias Felleisen. Contracts for firstclass modules. In Symposium on Dynamic Languages, pages 27-38, October 2009.

[45] T. Stephen Strickland and Matthias Felleisen. Nested and dynamic contract boundaries. In Implementation and Application of Functional Languages, September 2009.

[46] Clemens Szyperski. Component Software. Addison-Wesley, 1997. 